\title{
El modelo social como perspectiva de intervención desde el trabajo social en personas con la capacidad modificada
}

\section{The social model as a perspective of intervention from social work in people with modified capacity}

\section{Palabras clave:}

Discapacidad, modificación de la capacidad, modelo social, calidad de vida, apoyo social.

\section{Keywords:}

Disability, ability modification, social model, quality of life, social support.

\section{David González Casas <davgon14@ucm.es>}

Universidad Complutense de Madrid. España

\section{Esther Mercado García <emercado@ucm.es>}

Universidad Complutense de Madrid. España

\section{Introducción}

La realidad histórica y social de las personas con discapacidad ha ido variando de forma ostensible en función de los arquetipos presentes en la sociedad. La concepción otorgada a dicho grupo marca las líneas maestras de las acciones públicas dirigidas a implementar medidas que mitiguen las coyunturas adversas a las que se enfrentan de facto las personas con discapacidad.

Así, se ha pasado de un modelo de prescindencia a un modelo médico o rehabilitador y, en último lugar, a un modelo de autonomía e inclusión social que centra su análisis en el entorno, huyendo de facto de los postulados que articulan sus propuestas basándose en las deficiencias de las personas con discapacidad. La inclusión lleva implícito el impulso y reconocimiento de la esfera social, implementando nuevos modelos de actuación que incluyan el entorno como factor determinante, ya que es ahí donde se ve consolidada la exclusión (Jimenez, 2007).

Por lo tanto, el principal argumento teórico y analítico que refleja el mencionado modelo reside en conceptualizar, por primera vez en la historia, la discapacidad como un fenómeno social. Se vincula la discriminación sufrida por las personas con discapacidad, con un modelo social erróneo y/o inoperante que estandariza unos patrones conductuales que desligan al colectivo

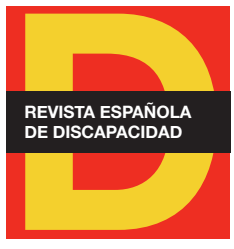

Para citar:

González, D. y Mercado, E. (2019): "El modelo social como perspectiva de intervención desde el trabajo social en personas con la capacidad modificada". Revista Española de Discapacidad, 7 (I): 241-249.

Doi: <https://doi.org/10.5569/23405104.07.01.12> 
de su total inclusión, es decir, se hace especial énfasis en la identificación de los marcos socio-culturales como una de las principales barreras a las que se enfrenta el colectivo, siendo dichas vicisitudes el objeto de remoción como única vía para la correcta inclusión del colectivo.

Sólo desde la perspectiva social es posible la conjunción de las personas con discapacidad y el respeto basado en los derechos, ya que el soslayo de su dignidad inherente como seres humanos no está ligado a ninguna vicisitud individual intrínseca que impida su desarrollo social y personal, sino al constructo social que inhabilita dicho desarrollo (Ospina, 2010).

\section{Plasmación del modelo social en la Convención Internacional sobre los Derechos de las Personas con Discapacidad}

La Convención Internacional sobre los Derechos de las Personas con Discapacidad fue aprobada por consenso en la Asamblea General de las Naciones Unidas el 13 de diciembre de 2006, y nace bajo el amparo de la necesidad palpable de dar un nuevo impulso respecto a la visión de las personas con discapacidad en la sociedad, y en cómo ésta se torna un factor determinante en los procesos excluyentes del colectivo.

Sin duda, la discapacidad no es un fenómeno estático, sino que su evolución se conjuga con las consideraciones sociales y jurídicas tendentes en cada momento, es decir, la concepción de las personas con discapacidad ha ido variando de forma ostensible a lo largo del tiempo, por lo que la Convención Internacional aparece como una herramienta más en el proceso de la equiparación de derechos y la erradicación de los procesos excluyentes, en los que se ven inmersos las personas que poseen algún tipo de discapacidad.

Tras la firma de la Convención por parte de veinte estados, cifra prevista en el Art.45 de la misma como requisito para la entrada en vigor del tratado, las personas con discapacidad ocupan un 'espacio' legislativo internacional propio que ahonda en la ruptura de los postulados caritativos y asistenciales anteriores, dando paso a un reconocimiento pleno de los derechos humanos vinculándolos a las personas con discapacidad. Por lo tanto, la Convención no 'crea' derechos específicos para el colectivo, sino que representa un instrumento jurídico que detalla cómo hacer efectivos dichos derechos humanos en referencia a la población a la que hacemos alusión.

Por ende, la Convención debe de ser entendida como un avance jurídico, ético y social en lo que respecta a la conceptualización de los derechos de las personas con discapacidad. Lo recogido en ella no sólo supone un hito histórico en lo concerniente al ámbito de la discapacidad, sino un elemento que representa la configuración de unos parámetros internacionales que abogan por la justicia social, dotando del impulso necesario para formar una sociedad más igualitaria, democrática y respetuosa con la dignidad intrínseca de todos los seres humanos. 
2.1. Igual reconocimiento como persona ante la ley y su implicación en los procesos de modificación de la capacidad

Dentro del abanico de disposiciones vinculadas a la igualdad dentro de la Convención Internacional, y tomando como referencia que las dificultades que se encuentran las personas con discapacidad para tomar decisiones sobre su esfera personal en ocasiones pueden estar motivadas por las barreras existentes en el entorno social (Cuenca, 2012), el artículo 12 señala la necesidad de remover o eliminar cualquier obstáculo que dificulte el acceso del colectivo al disfrute y reconocimiento de su plena capacidad, instando a adaptar los procesos relativos a la determinación de la capacidad recogidos en los ordenamientos nacionales, a la idiosincrasia única y personal de cada sujeto.

Así, en el artículo mencionado se cita: 1." Los Estados Partes reafirman que las personas con discapacidad tienen derecho en todas partes al reconocimiento de su personalidad jurídica. 2. Los Estados Partes reconocerán que las personas con discapacidad tienen capacidad jurídica en igualdad de condiciones con las demás en todos los aspectos de la vida. 3. Los Estados Partes adoptarán las medidas pertinentes para proporcionar acceso a las personas con discapacidad al apoyo que puedan necesitar en el ejercicio de su capacidad jurídica".

Mediante lo expuesto, la Convención hace especial hincapié en la capacidad que poseen todas las personas con discapacidad para disfrutar y ejercer sus derechos, y en especial, aquellas que hasta el momento parecían haber quedado al margen de todas las regulaciones internacionales y nacionales, es decir, las personas con discapacidad intelectual o enfermedad mental (Barranco et al., 2012).

Frente al paradigma médico, el modelo social sobre el que se asienta la Convención, y en concreto el citado artículo, pone de relieve la necesidad de replantearse la consideración jurídica que albergan en los ordenamientos nacionales e internacionales las personas con discapacidad. La práctica habitual consistía en la escasa o nula consideración acerca de las capacidades de este colectivo, por lo que las actitudes paternalistas o benefactoras presidían el pensamiento social y jurídico. La Convención hace patente la necesidad de instaurar un sistema que abogue por una verdadera igualdad en el goce y acceso a los derechos, incluida la plena capacidad (en el caso de España, debemos referirnos a la capacidad jurídica y a la de obrar), modificando las condiciones planteadas anteriormente y resaltando la importancia de proteger y poner en valor las capacidades inherentes al colectivo, con el objetivo de favorecer un óptimo desarrollo personal y social.

En la misma línea, no corresponde a las personas con discapacidad 'demostrar' su valía para disfrutar de una plena capacidad de obrar, sino que es el espacio social el que debe amoldarse a las circunstancias personales de las personas insertas en el colectivo (Barranco et.al., 2012). Lo expuesto hace referencia a las situaciones en las que se asocian las discapacidades intelectuales a una nula capacidad para autogobernarse de forma eficiente; este enfoque errático debe de ser sustituido por nuevos planteamientos que insten a la sociedad y a las políticas públicas a implementar herramientas, que busquen adecuar las condiciones sociales que dificultan el acceso al disfrute de la plena capacidad del colectivo. Para ello, la propia Convención recoge algunos mecanismos a tener en cuenta: la accesibilidad universal (adecuación de espacios públicos, adaptación de medios de comunicación, información accesible, etc.) y los apoyos individualizados.

La Convención plantea unos cambios sustantivos en el marco jurídico referente a los procesos de modificación de la capacidad; se trata de remover los obstáculos presentes, que dificultan la toma de decisiones 
propias de las personas con discapacidad. $Y$ donde existan condiciones personales que dificulten el autogobierno de las mismas se debe proceder a implementar los ajustes necesarios -puede ser el apoyo de un tercero- que faciliten la toma de decisiones propias (International Disability Alliance, 2018).

Cuando las medidas relativas a la accesibilidad universal no sean suficientes para que la persona con discapacidad goce de una completa igualdad en el acceso a su capacidad, se implementarán apoyos individualizados y amoldados a las características intrínsecas de cada persona. Dichos apoyos -intervención de un tercero-, cuentan con los límites expuestos en el apartado 4 del artículo 12, que refiere: "Los Estados Partes asegurarán que en todas las medidas relativas al ejercicio de la capacidad jurídica se proporcionen salvaguardias adecuadas y efectivas para impedir los abusos de conformidad con el derecho internacional en materia de derechos humanos. Esas salvaguardias asegurarán que las medidas relativas al ejercicio de la capacidad jurídica respeten los derechos, la voluntad y las preferencias de la persona, que no haya conflicto de intereses ni influencia indebida, que sean proporcionales y adaptadas a las circunstancias de la persona, que se apliquen en el plazo más corto posible y que estén sujetas a exámenes periódicos por parte de una autoridad o un órgano judicial competente, independiente e imparcial. Las salvaguardias serán proporcionales al grado en que dichas medidas afecten a los derechos e intereses de las personas".

Como se desprende de lo citado en el apartado cuarto, las medidas deben perseguir el apoyo en la toma de decisiones, respetando la voluntad y preferencias de la persona a la que se le brinda dicho apoyo y, salvo en casos excepcionales, nunca fundamentadas en la sustitución total de la voluntad del interesado.

Es decir, la Convención plantea nuevas pautas a seguir en lo concerniente a la respuesta judicial en materia de limitaciones, problemáticas o dificultades para el autogobierno, abandonando el sistema de representación legal, en la que la voluntad del incapaz era sustituida por el tutor, para adentrarse en un sistema de apoyos puntuales y concretos, que respeten su capacidad natural para la toma de decisiones (Ladrón, 2012).

La capacidad de obrar no puede ser cuestionada simplemente por la existencia de una discapacidad psíquica o una enfermedad mental, hecho que vulneraría de facto el artículo 12 de la Convención Internacional, y que rompe con el status clásico que asociaba ciertas discapacidades con procesos de incapacitación total (Barranco et.al., 2012). En consecuencia, se debe de analizar, además de las condiciones individuales de cada sujeto, las características sociales y ambientales, con el fin de diseñar los apoyos necesarios que precise en el ejercicio de su capacidad (Cuenca, 2012).

En la actualidad se encuentra en proceso de trámite una reforma del Código Civil que pretende adaptar el sistema de modificación de la capacidad a los preceptos recogidos en la Convención Internacional sobre los derechos de las personas con discapacidad.

3. El apoyo social como herramienta referencial en la práctica del trabajo social respecto a las personas con la capacidad modificada

Tomando como referencia teórica lo especificado hasta el momento, parece oportuno estimar que las prácticas destinadas a la inclusión plena y efectiva de las personas con discapacidad con la capacidad de obrar 
modificada deben pivotar sobre uno de los constructos fundamentales del modelo social de la discapacidad: erradicar los obstáculos de índole social presentes en los espacios individuales y comunitarios en los que desarrollan su proyecto vital las personas con discapacidad (De Lorenzo y Palacios, 2007).

El trabajo social debe tornarse en el referente/abanderado de dichos postulados, ya que la propia idiosincrasia de la disciplina lleva asociada la construcción de espacios personales y sociales que permitan desarrollar de una forma óptima el proyecto vital establecido por cada individuo.

Hasta la fecha y, pese a los ostensibles avances observados en las prácticas relacionadas con el ámbito de la discapacidad y, en concreto, con personas con la capacidad modificada, seguimos observando actitudes de índole paternalista o proteccionista que dificultan, mitigan y sesgan el desarrollo de las capacidades del colectivo para decidir sobre sus propias vidas. Estas actitudes, fundamentadas en la pseudo creencia de la incapacidad de las personas para tomar decisiones adecuadas sobre su propia vida, deslegitiman el verdadero sentido de la intervención profesional. Como refería Perske (1972:197), "hay dignidad en tomar un riesgo y puede haber una indignidad deshumanizadora en la seguridad", es decir, para que una persona pueda desarrollar una vida plena, ineludiblemente, debe asumir espacios de fracaso, riesgo, equivocaciones, frustraciones, etc. En ese espacio de "aprendizaje a través de la experiencia"1, el trabajador social o la trabajadora social debe ser el referente profesional que articule los mecanismos precisos para facilitar un correcto 'engranaje' persona-entorno.

\subsection{El apoyo social como instrumento de inclusión}

Pese a la existencia de una multiplicidad de autores (Cassel, 1976; Turner y Tenhoor, 1978; Caplan, 1974) que han establecido una definición propia del apoyo social, es Nan Lin (1986) el que trata de aglutinar, en base a los aspectos teóricos más relevantes, una definición holística del término. Según el citado autor el apoyo social debe de ser entendido como "las provisiones expresivas o instrumentales -percibidas o recibidas- proporcionadas por la comunidad, las redes sociales y las personas de confianza, tanto en situaciones de crisis como cotidianas (Lin, 1986:19)".

En el ámbito de la investigación e intervención social existe una posición arraigada que concibe el bienestar psicosocial como un constructo en continuo equilibrio entre los estresores presentes y los recursos disponibles (Gracia et al., 2002). Dichos fenómenos deben entenderse como dos magnitudes contrapuestas que actúan sobre el bienestar de cualquier sujeto (individual o social).

En base a lo anterior, y para seguir delimitando las implicaciones del apoyo social en el bienestar psicosocial de cualquier individuo, debemos conocer qué determinantes causales del apoyo social están intrínsecamente ligados al constructo (Sarason, 1990). Dichos determinantes son -o deben ser- los ejes estructurales de las prácticas profesionales que pretendan fomentar un correcto y, por ende, funcional apoyo social en las personas con discapacidad con la capacidad de obrar modificada:

1. Así lo denomina Joaquín María Rivera en "Notas sobre el apoyo a la autodeterminación de la persona con discapacidad (incapacitada) en el Código Civil a partir de la CIDAD”, en La encrucijada de la incapacitación y la discapacidad, 2011, pp.347-372. cuando hace referencia al Art.26 de la CIDAD (Habilitación y rehabilitación). 
1. Determinantes intrapersonales. En este espacio nos encontramos con modelos cognitivos de representación del self, la autoestima, las habilidades sociales y las características de la/s persona/s proveedoras de apoyo.

2. Determinantes interpersonales. Aspectos como la intimidad, reciprocidad y las características estructurales de la red social, son los elementos clave a tener en cuenta en el ejercicio profesional.

3. Determinantes situacionales. En esta esfera o campo de trabajo/estudio, se deben valorar las situaciones estresantes como factor clave en la cantidad y calidad del apoyo social percibido y recibido.

4. Determinantes macrosociales. Indudablemente la posición o jerarquía social de un individuo o grupo se tornan como procesos determinantes a la hora de implementar actuaciones profesionales dirigidas a incrementar los niveles de apoyo social (Gracia et al., 2002)².

Habitualmente las investigaciones o prácticas insertas en el campo de la discapacidad, tienden a entender el apoyo social desde una perspectiva estructural, es decir, como una herramienta de análisis que estudia el tamaño y cantidad de las redes de apoyo. Se torna innegable que el análisis de las estructuras de las redes sociales es una pieza fundamental en la investigación e intervención desde el prisma del apoyo social. Ahora bien, no debemos caer en el error de sesgar la subjetividad intrínseca en cualquier proceso de índole social. Partir de la premisa de que todas las relaciones sociales impulsan en igual medida el bienestar del individuo sería simplificar nuestro análisis de facto. Lo expresado encuentra su complemento desde la perspectiva funcional del apoyo social, que se centra en la calidad de las citadas redes desde tres prismas definidos (Gracia et al., 2002):

1. Apoyo emocional.

2. Apoyo de información.

3. Apoyo material o tangible

La conjugación de estas dos esferas -estructural y funcional- debe ser el vehículo articulador de toda práctica profesional en el campo del apoyo social. Más allá de incrementar las redes sociales del sujeto, debemos fomentar la calidad de las interacciones sociales. En este sentido, Gerald Caplan nos conduce a realizar una estratificación de ostensible relevancia:

- Apoyo recibido. Se trata de las transacciones reales de apoyo que se producen entre la persona y su entorno.

- $\quad$ Apoyo percibido. Nos referimos a las percepciones del sujeto respecto a sus relaciones sociales (Caplan, 1974).

En la práctica, el trabajo social se ha fundamentado en estudiar e intervenir sobre el apoyo recibido, es decir, se ha encorsetado en una función asistencialista que dirimía la cantidad de apoyos tangibles. Las nuevas prácticas deben permutar hacia la visión subjetiva del interesado respecto al nivel y calidad del apoyo social que recibe. La relevancia de dicho constructo reside en que, dentro de la propia valoración subjetiva del individuo, podemos encontrar las claves del apoyo social a trabajar y, además, la propia esencia del apoyo

2. En un estudio realizado por Gracia et al. en el año 1995, en el que los autores examinaron el nivel de apoyo social en función del estatus socioeconómico, se determinó que el tamaño de la red social, la afiliación a organizaciones y el contacto con otros miembros de la red, tienden a ser mayores a medida que se incrementa el nivel socioeconómico. 
social reside en la percepción de la propia persona sobre la disponibilidad de recursos de apoyo llegado el caso de necesitarlos -más si cabe en personas con la capacidad modificada-. De esta forma y, como señalan Gracia et al. (2002: 28), "la experiencia personal del peligro que entraña cualquier situación depende, más que de las características del evento exclusivamente, de la valoración que sobre el evento realiza el individuo". Desde el trabajo social debemos fomentar que las personas con discapacidad con la capacidad de obrar modificada adquieran las habilidades necesarias para generar espacios de percepción de apoyo positivos que conlleven la mejora en la capacidad de afrontamiento de situaciones difíciles y, por ende, una herramienta eficaz en la construcción de un proyecto vital fundamentado en las propias preferencias y/o necesidades del sujeto.

Las personas con discapacidad, y en concreto, las personas con la capacidad modificada, mantienen una cuasi perenne situación de exclusión social o relacional y esto es debido fundamentalmente a que han sido situadas por la sociedad estandarizada en un espacio social especialmente creado para ellas, donde no resultan sujetos plenos de derecho (Pindado, 2015). Una de las principales secuelas derivadas de dicha consideración es la creación de redes de apoyo ineficientes, fundamentadas en la sustitución o sobreprotección en la toma de decisiones propia. Es decir, se torna determinante contar con la percepción del colectivo sobre sus propios acontecimientos personales y sociales y, en base a ello, el trabajo social debe fomentar un apoyo social eficiente, desde un punto de vista estructural y funcional, en el que prevalezca y sea determinante la visión subjetiva del colectivo sobre sus propias necesidades. Para abarcar todo el espectro -micro, meso y macro- del apoyo social, el trabajo social tiene legitimidad metodológica para implementar acciones destinadas a incidir en todas las dimensiones citadas, con el afán de repercutir en la mejora de la calidad de vida de las personas con la capacidad de obrar modificada, respetando su autonomía y dotando a la intervención de un carácter resiliente y de empoderamiento, fundamentada en la búsqueda de los apoyos necesarios para un ejercicio pleno de sus derechos en condiciones de igualdad respecto al resto de la ciudadanía.

Para ejemplarizar lo citado hasta el momento, debemos de repensar la figura del trabajador social en la atención a personas con la capacidad modificada como un agente que implemente actuaciones en las que se estudien, evalúen y respeten los gustos y preferencias del sujeto, sus metas y objetivos; diseñando planes individualizados en los que se establezcan los apoyos necesarios para facilitar el acceso a una vida acorde a las necesidades objetivas y subjetivas del propio sujeto.

\section{Conclusiones}

En la actualidad y pese a que desde la esfera doctrinal y/o investigadora nos encontramos con estudios que aluden al modelo social como enfoque primario en el trabajo con el colectivo formado por personas con discapacidad, la realidad nos revela que aún perdura en las estructuras sociales y culturales una visión excluyente fundamentada en percepciones paternalistas, y por ende, marginadoras. El ejemplo de lo citado se hace palpable en las medidas adoptadas en los procesos de modificación de la capacidad, en los que aún hoy en día se torna necesario la implementación plena de un sistema fundamentado en los apoyos y no en la sustitución de la capacidad de obrar por parte de un tercero. 
Tomando como referencia la doctrina del modelo social -marco referencial en los ordenamientos jurídicos desde la aprobación de la Convención Internacional sobre los derechos de las personas con Discapacidad-, desde el trabajo social debemos abanderar las acciones investigadoras y profesionales destinadas a erradicar los obstáculos sociales causantes del inadecuado acceso del colectivo al disfrute de una vida lo más independiente y autónoma posible. Las relaciones entre el profesional y el sujeto, además de horizontales, deben primar la creación de mecanismos que, a través del apoyo social, fomenten la inclusión social mediante el estudio y conocimiento de las necesidades y objetivos personales de las personas con la capacidad modificada, y por ende, establezcan un sistema de apoyos que aumente la percepción de control sobre sus propias vidas. 
Referencias bibliográficas

Barranco, M. et al. (2012): "Capacidad jurídica y discapacidad: el artículo 12 de la Convención de Derechos de las Personas con Discapacidad". Anuario de la Facultad de Derecho, 5: 53-80.

Caplan, G. (1974): Supports systems and comunity mental health: Lectures on concept development. Nueva York: Behavorial Publications.

Cassel, J. (1976): "The contribution of the social enviroment to host resistance". American journal of epidemiology, 104: 107-123.

Cuenca, P. (2012): "El sistema de apoyo en la toma de decisiones desde la Convención Internacional sobre los Derechos de las Personas con Discapacidad: principios generales, aspectos centrales e implementación en la legislación española". Revista electrónica del Departamento de Derecho de la Universidad de La Rioja, 10: 71-74.

De Lorenzo, R. y Palacios, A. (2007): “Discapacidad, derechos fundamentales y protección constitucional”, en Laorden, J. y Terreros, J. L. (coords.): Los derechos de las personas con discapacidad. Madrid: Consejo General del Poder Judicial.

Gracia, E. et al. (2002): Evaluación de recursos y estresores psicosociales en la Comunidad. Madrid: Sintesis.

International Disability Alliance (2010): Principios para la implementación del artículo 12 de la CDPD (en línea). <http://www.internationaldisabilityalliance.org/es/node/213>, acceso 15 de junio de 2018.

Jiménez, A. (2007): "Conceptos y tipologías de la discapacidad: documentos y normativas de clasificación más relevantes", en De Lorenzo, R. y Pérez, L. C. (coords.): Tratado sobre discapacidad. Madrid: Thomson Reuters Aranzadi.

Ladrón, M. P. (2012): "Los procesos en materia de capacidad a la luz de la Convención de Naciones Unidas de Derechos de las Personas con Discapacidad". Anuario de la Facultad de Derecho, 5: 203-222.

Lin, N. (1986): “Conceptualizing social support”, en Lin, N. et al. (eds.): Social Support, Life Events, and Depression. Nueva York: Academic press.

Ospina, M. (2010): “Discapacidad y Sociedad Democrática”. Revista Derecho del Estado, 24, 143-164.

Perske, R. (1972): "The dignity of risk", en Wolfensberger, W. P. (. En The principle of normalization in human services (págs. 194-200). Toronto: National Institute on Mental Retardation.

Pindado, F. (2015): Hacia una única catalogación de los Derechos Fundamentales - Los derechos económicos, sociales y culturales de las personas con discapacidad como derechos fundamentales-. Madrid: CINCA.

Rivera, J. M. (2011): "Notas sobre el apoyo a la autodeterminación de la persona incapacitada en el Código Civil a partir de la convención de N.U. sobre derechos de las personas con discapacidad", en Pérez de Vargas, J. y Pereña, M. (coords.): La encrucijada de la incapacitación y la discapacidad. Madrid: Wolters Kluwer.

Sarason, I. (1990): "Social support and interactional proceses: A triadic hypothesis". Journal of Social and Personal Relationship, 7: 495-506.

Turner, J., y Tenhoor, W. (1978): "The NIMH Community Support Program: Pilot approach to a needed social reform". Schizophrenia Bulletin, 4: 319-348. 